\title{
Review Article \\ The Effectiveness of Lifestyle Adaptation for the Prevention of Prediabetes in Adults: A Systematic Review
}

\author{
George Kerrison, ${ }^{1}$ Richard B. Gillis, ${ }^{1,2}$ Shahwar I. Jiwani, ${ }^{1,2}$ Qushmua Alzahrani, ${ }^{1,2}$ \\ Samil Kok, ${ }^{2,3}$ Stephen E. Harding, ${ }^{2}$ Ian Shaw, ${ }^{4}$ and Gary G. Adams ${ }^{1,2}$ \\ ${ }^{1}$ School of Health Sciences, Faculty of Medicine South Block, Queen's Medical Centre, University of Nottingham, \\ Nottingham NG7 2HA, UK \\ ${ }^{2}$ University of Nottingham, Sutton Bonington Campus, NCMH Building, Sutton Bonington, Leicestershire LE12 5RD, UK \\ ${ }^{3}$ Department of Food Engineering, Faculty of Engineering \& Architecture, Abant İzzet Baysal University, Gölköy, 14280 Bolu, Turkey \\ ${ }^{4}$ Faculty of Medicine and Health Sciences, University Park, University of Nottingham, Nottingham NG7 2RD, UK
}

Correspondence should be addressed to Gary G. Adams; gary.adams@nottingham.ac.uk

Received 10 November 2016; Accepted 26 February 2017; Published 16 April 2017

Academic Editor: Samy McFarlane

Copyright (C) 2017 George Kerrison et al. This is an open access article distributed under the Creative Commons Attribution License, which permits unrestricted use, distribution, and reproduction in any medium, provided the original work is properly cited.

\begin{abstract}
Diabetes prevalence is increasing exceptionally worldwide and with this come associated healthcare costs. The primary outcome of this systematic review was to assess glycaemic control and incidence of Type 2 diabetes mellitus (T2DM) diagnosis after exercise and dietary intervention (measured with any validated scale). The secondary outcome assessed body mass index change, weight change, and physical exercise capacity after diet and exercise intervention (measured with any validated scale). 1,780 studies were identified from searching electronic databases. Relevant studies went through a selection process. The inclusion criteria for all studies were people with prediabetes diagnosed by either impaired glucose tolerance (IGT) or impaired fasting glucose (IFG). Lifestyle adaptation reduced the incidence of diabetes development more than standard treatment. Furthermore, better glycaemic control, improved physical exercise capacity, and increased weight reduction were observed with lifestyle intervention over standard treatment. Finally, improvements over the long term deteriorated, highlighting problems with long-term adherence to lifestyle changes. Overall, cumulative incidence of diabetes is drastically reduced in the intervention groups compared to control groups (standard care). Furthermore, glycaemic control was improved in the short term, with many participants reverting to normoglycaemia.
\end{abstract}

\section{Introduction}

Cumulatively, all subcategories of diabetes mellitus affect approximately 382 million people worldwide, increasing to an estimated 592 million by 2035 [1], emphasising the global burden of diabetes. In UK, 3.2 million people have been diagnosed with diabetes; a conservative estimate predicts an increase to 5 million by 2025 [2], which equates to one in seventeen people in UK or prevalence of $6.0 \%$ [3]. In $2010 / 2011$, the total cost to UK for diabetes was $£ 23.7$ billion, with $£ 8.8$ billion directly in T2DM costs, and total cost is predicted to rise to $£ 39.8$ billion by 2035 [4]. $10 \%$ of the NHS budget is estimated to be spent on diabetes $[4,5]$.
Prediabetes is a relatively recent medical term, which raises many issues for debate [6]. The American Diabetes Association [7] endorses the term, but both WHO [8] and NICE [6] oppose it. The National Institute for Clinical Excellence (NICE) considered that impaired glucose regulation (IGR) should be used instead of prediabetes; inevitable progression to diabetes is implied using prediabetes [6]. Diabetes UK (2009) states that impaired fasting glycaemia (IFG), IGT, and glycosylated haemoglobin (HbAlc) should be designated for usage between healthcare professionals, with prediabetes used for the lay person. The ADA combined IGT and IFG into the blanket term prediabetes. In 2003, the ADA lowered the threshold for IFG and HbAlc against 
advice from other healthcare bodies [9]. Threshold lowering was justified by needing earlier detection to reduce diabetes development and cardiovascular disease [10]. Arguments against the threshold change included consequences with life insurance, possible discrimination, and causing anxiety about developing diabetes when it might not occur [11]. Cut-off points for prediabetes are arbitrary with no biological basis for the test values [12].

The Da Qing IGT and Diabetes Study was one of the original studies on prediabetes [13] and involved 577 participants with IGT who underwent exercise and diet treatment or diet only or exercise only. Follow-up was at 2 years and 6 years to identify any participants who had developed T2DM. At 6 years, $67.7 \%$ of the control group (standard treatment) developed T2DM compared with $46.0 \%$ in the diet plus exercise group. It was assessed that the diet plus exercise intervention decreased the risk of developing diabetes by $42 \%$. While this study was conducted nearly two decades ago, it highlights that prediabetes has always been present and can potentially be treated with diet plus exercise more effectively than the standard treatment delivered.

Oldroyd et al. [14] evaluated lifestyle modification for improving health in individuals with IGT. 39 intervention participants were encouraged to eat more fruit and vegetables, reduce fat and sugar intake, and increase dietary fibre. Furthermore, they were encouraged to achieve 20-30 minutes of aerobic activity at least once a week. At 24 months, (after commencement of study), improvements in $2 \mathrm{hr}$ plasma glucose were not significantly different between the control and intervention groups. However, more participants in the intervention group compared to the control group reverted to normoglycaemia with $20 \%$ versus $13 \%$ at 24 months.

A systematic review by Orozco et al. [15] included 8 randomised controlled trials, with a total of 2241 participants using exercise plus diet treatment, compared to 2509 participants undergoing standard treatment. Interventions included calorie restriction diets that were low in fat and high in carbohydrates and fibre. Exercise interventions varied but mainly consisted of 150 minutes a week of brisk walking or other activities. The interventions were delivered by physiotherapists and dieticians across the board. On average, the incidence of diabetes reduced by $37 \%$ with exercise and diet. There were also favourable effects for body weight, waist circumference, and blood pressure. However, results of this study should be interpreted with caution as the results are now 7 years old and healthcare advances rapidly.

In 2009, Lindahl et al. tested 168 individuals for diabetes incidence, diet improvement, and exercise capacity [16]. 83 underwent 1-month intensive lifestyle intervention staying at a wellness centre where they underwent overall 140 hours of scheduled activities and had healthy meals prepared for them; 85 underwent standard treatment at home. At the 1-year follow-up, a 70\% decrease in progression to T2DM was seen in the intervention group. However, at 5 years, most of the beneficial effects of the intervention had depleted; exercise capacity was one of the only outcomes which stayed at an appropriate level. This study highlights the fact that there is a maintenance problem with continuing lifestyle adaptations.

The primary outcome of this systematic review was to assess glycaemic control and incidence of T2DM diagnosis after exercise and dietary intervention. The secondary outcome assessed body mass index change, weight change, and physical exercise capacity after diet and exercise intervention (both measured with any validated scale).

\section{Methods}

In total, 1,780 studies were identified from searching the electronic databases CINAHL $(n=62)$, MEDLINE $(n=$ 226), EMBASE $(n=554)$, PsycINFO $(n=19)$, and Cochrane CENTRAL $(n=919)$ (Figure 1). Additional searching of reference lists from recent relevant systematic reviews and hand searching of studies identified 4 further studies. Results from the search were imported to Endnote X7 for assessment of relevance to this review. 271 duplicates and 30 studies not in the English language were removed. The remaining studies were assessed by title alone and 1,360 studies were removed. For the remaining studies, the abstract was assessed, with 104 studies deemed irrelevant. The full texts of the remaining 19 studies were examined against the inclusion and exclusion criteria for the review. 10 studies were excluded after full-text assessment. The remaining 9 studies met the inclusion criteria for the review (Table 1). All assessments were completed twice by different authors.

\section{Excluded Studies}

Of the 19 studies that had full-text review, 10 studies were excluded for not meeting the inclusion criteria. These studies were excluded for the following reasons: being unable to gain access to full text $(n=2)$, pilot for an intended RCT $(n=1)$, RCT but prediabetes is not in the inclusion criteria $(n=3)$, review of previous study $(n=1)$, mixed intervention $(n=$ $1)$, glycaemic control not an outcome $(n=1)$, and not for specified period of 8-week intervention and not compared with normal treatment $(n=1)$.

\section{Risk of Bias in Included Studies}

The methodological property of all the included studies was assessed for risk of bias (Figure 2). The assessment involved using the Cochrane collaborations tool for assessing risk of bias. The tool consists of 7 areas where bias could possibly be introduced and making judgements to assess if bias is introduced or not. This is completed by answering prespecified questions, for each of the 7 domains, and answering with either "yes" to indicate low risk or "no" to indicate high risk. "Unclear" indicates that too few details are available to make a judgement on risk of bias. The final domain, other biases, should be used to assess the study as a whole for its risk of bias. Collected data was inputted to RevMan 5.3.

All of the studies included in this review were randomised control trials (RCTs). Critical appraisal of the studies included 


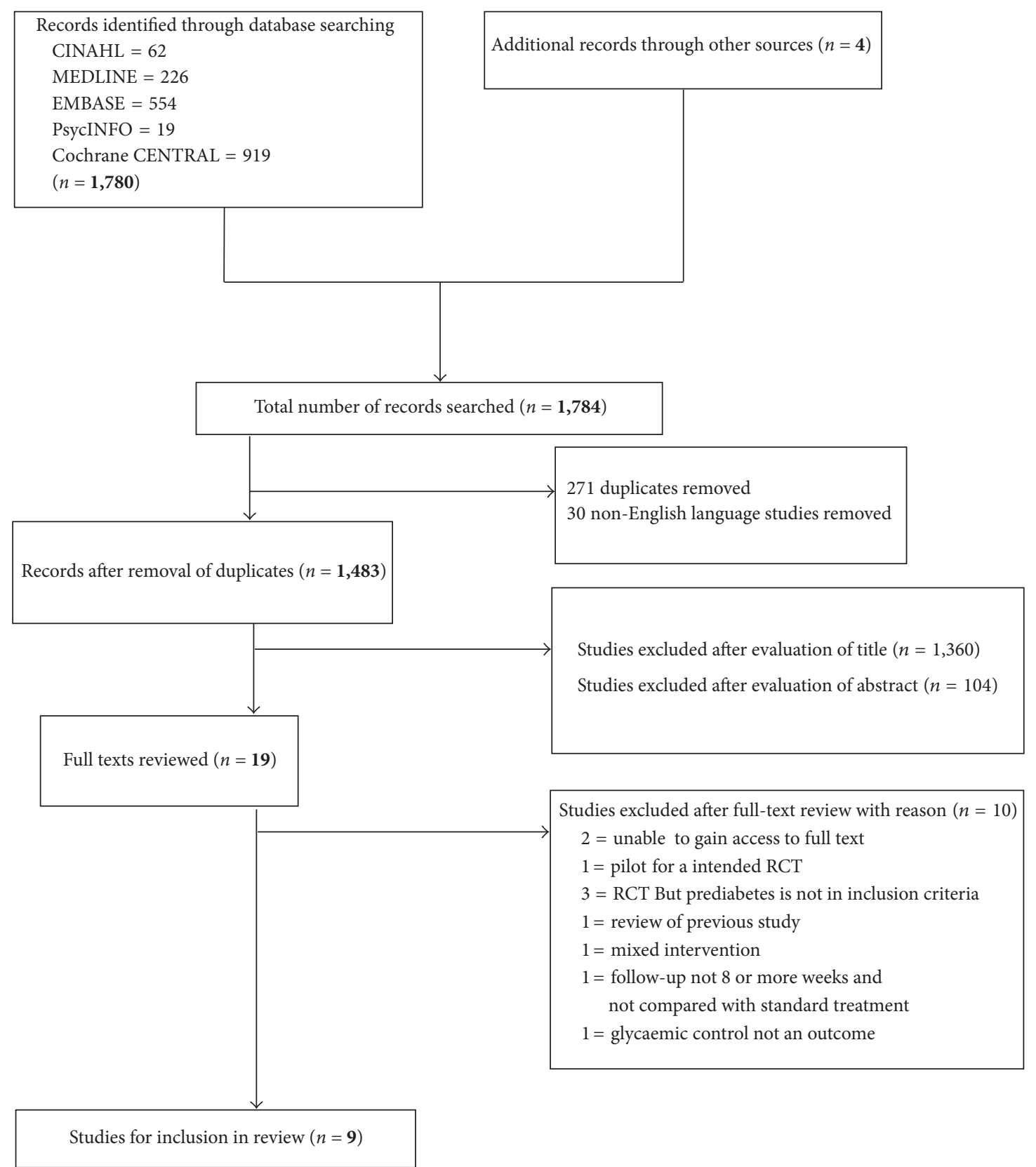

FIGURE 1: Flow chart of identification of included studies.

in this review showed that the methodological quality was high to moderate for all the studies. None of the studies included a high level of bias in their design. As such, all studies were included in the review.

Random assignment to either a control or intervention group was accomplished to a low risk in 6 of the studies by randomisation lists prepared by a third party, computer generated randomisation, or random allocation tables [18$21,24,25]$.

Allocation concealment was low risk in 2 studies [20, 24], where telephone calls or numerical values were given to denote the study group. One study was high risk [21], where participants had knowledge of their allocation to the intervention group. Six studies were lacking information to make a decision on allocation concealment [17-19, 22, 23, 25]. Due to the nature of the intervention, diet, and exercise, causing changes to the participants' normal lifestyle, it is difficult to conceal allocations. Therefore, this category will not carry a large weighting in terms of selection bias for this review.

Two studies stated that they blinded participants and personnel $[17,24]$. For 3 of the studies, it was unclear if the participants and personnel were blinded $[20,23,25]$. The remaining 4 studies did not blind participants or personnel $[19,22]$, with 2 studies specifically stating that participants are told what the intended intervention is for $[18,21]$. 


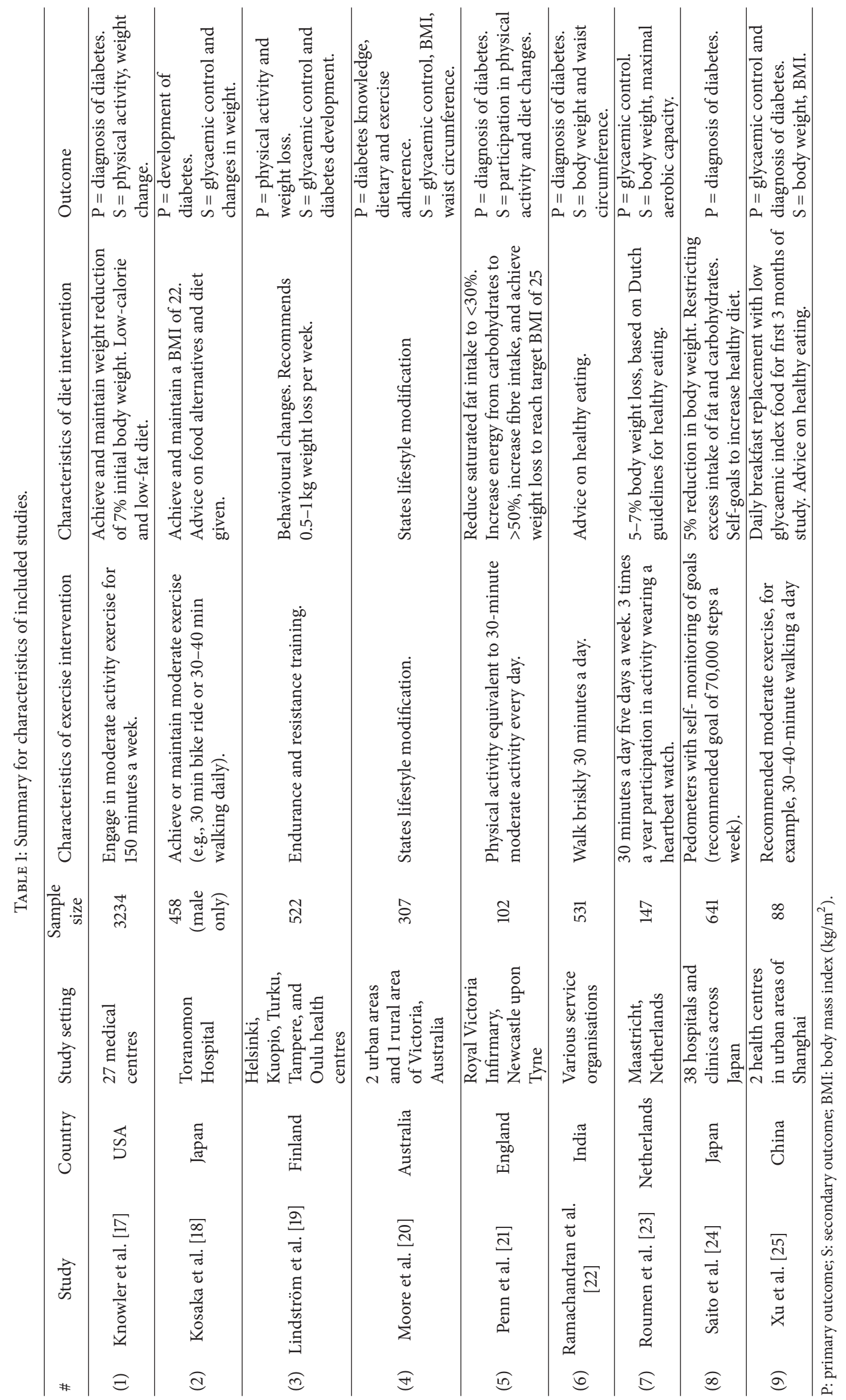




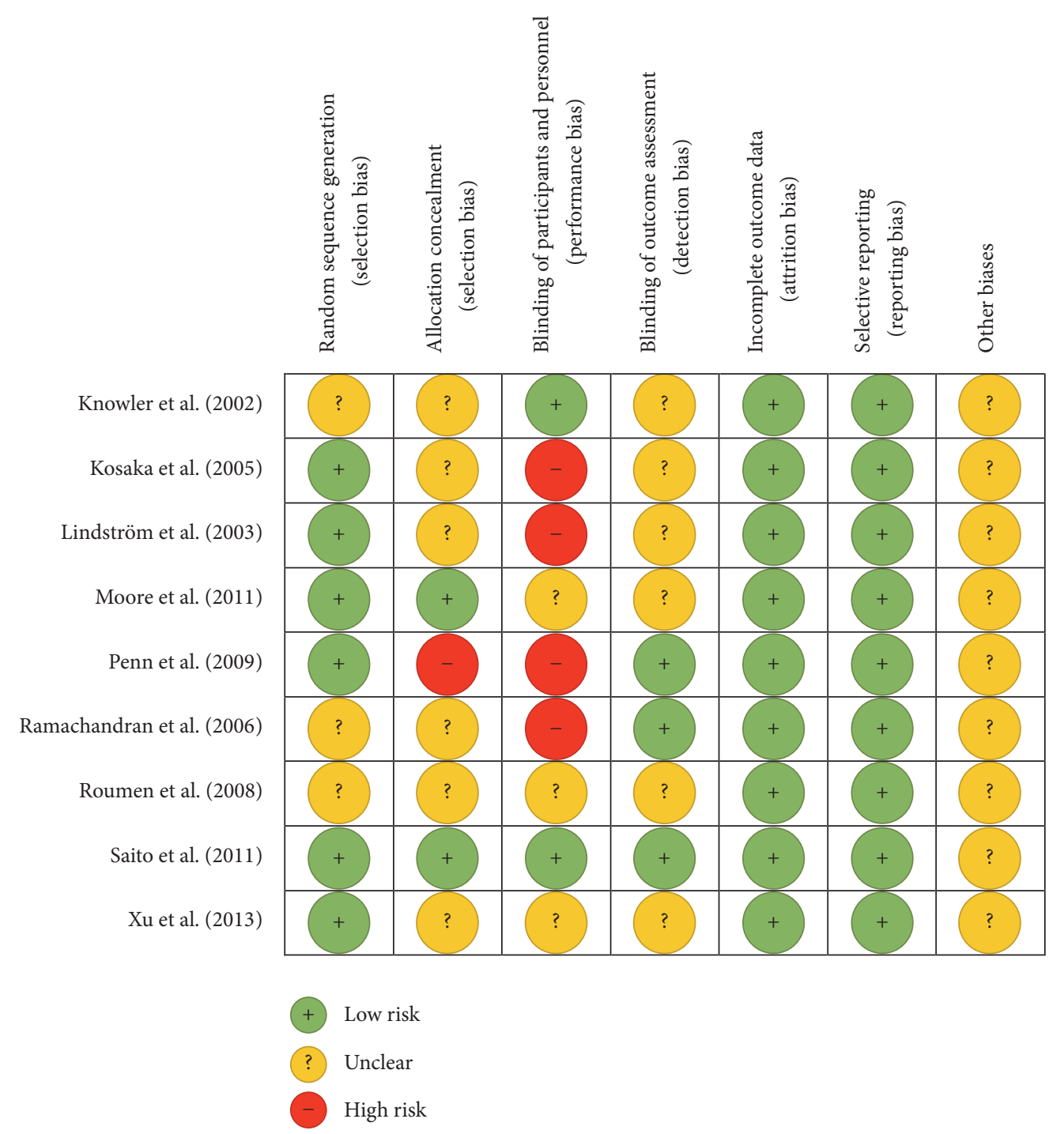

FIgURE 2: Risk of bias summary for included studies (produced in RevMan 5.3, 5 January 2015).

Blinding of outcome assessment was apparent in 3 studies $[21,22,24]$. It was unclear in the remaining 6 studies whether the outcome assessors were blinded or not [17-20, 23, 25].

All of the studies had a low risk value for both incomplete outcome data and selective reporting. For other biases, it is unclear due to the limited information available on the studies. However, it is apparent that all studies maintained a similar control group and an intervention group and no results were missing from the final reports.

The country of origin for the studies varies widely. Two studies are from Japan [24] and Kosaka et al., 2002, and each of the following countries was represented by one study 1 : USA [17], Finland [19], Australia [20], England [21], India [22], Netherlands [23], and China [25].

The majority of studies for exercise intervention recommended achieving and maintaining either 150 minutes of exercise a week or 30 minutes of exercise a day [17, 18, 21$23,25]$. For the remaining 3 studies, one utilised a pedometer and recommended 70,000 steps a week [24], one encouraged participants to take part in endurance and resistance training [19], and one stated "lifestyle modification" [20]. Diet interventions were targeted at motivating, encouraging, and enabling participants to achieve and maintain a target BMI or weight reduction. In one study, low glycaemic index meal replacement was used [25] and two studies did not state a target weight/BMI but provided healthy living advice [20,22].

The delivery method for the interventions varied from questionnaires to meetings with trained medical staff, motivational phone calls, and group based sessions. All of these were for a defined period of time. For the study's outcomes, the majority of studies used the diagnosis/prognosis of diabetes as the primary or secondary outcome $[17-19,21$, $22,24,25]$. Two studies did not specifically use diagnosis of diabetes as an outcome; these studies, as well as some others, focused on plasma glucose concentration [20, 23]. Other outcomes from the studies included changes in BMI, waist circumference, physical activity capacity, calorie intake, and diabetes knowledge. 


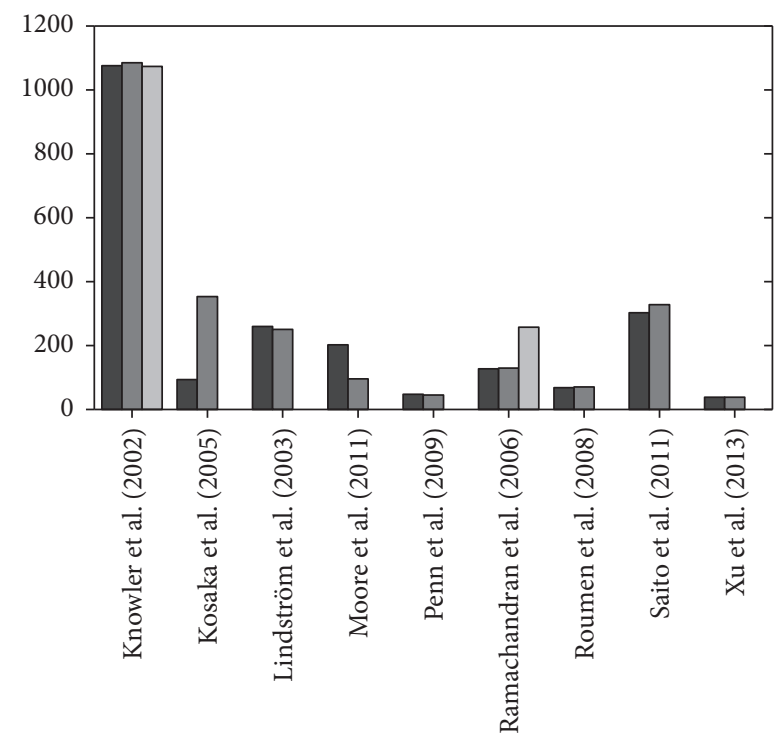

Intervention

Control

Pharmacological intervention participants

FIGURE 3: Sample size of included studies.

\section{Characteristics of Participants}

The characteristics of participants are summarised in Table 2. The study sample size (adjusted by removal of pharmacological interventions) ranged from 88 to 2,161 in size, with overall 4,695 participants involved in either lifestyle intervention or control groups (Figure 3 ). The total participants were 6,022 with pharmacological arms of studies included: 249 in Ramachandran et al. [22] and 1,078 in Knowler et al. [17]. Figure 4 expresses the breakdown for intervention, control, and pharmacological intervention participants for relevant studies.

All of the studies included both male and female participants. Apart from Kosaka et al's study [18] that had male participants only, it was acknowledged in the text that a previous study completed by the researcher included both males and females but there was a high dropout rate for female participants so male-only study was completed this time. The study by Ramachandran et al. [22] stipulated that only Asian Indian participants would be included in the study; all other studies were open to all ethnicities; however, coincidentally some only had one ethnicity included. The mean age of participants ranged between $46.1 \pm 5.7$ and $62.5 \pm$ 10.1. The mean body mass index (BMI) of participants ranged from $23.8 \pm 2.1$ to $34.1 \pm 5.5$. It should be noted that 4 of the studies are from Asian countries that have a lower BMI cut-off for BMI being recognised as a risk factor for diabetes; hence, some studies have a low BMI at baseline.

The inclusion criteria for all studies were prediabetes diagnosed by either IGT [17, 19-23] or IFG [18, 24, 25]. All studies, apart from Lindström et al.s study [19], stated a numerical value for diagnosis of prediabetes. The study by Lindström et al. states that prediabetes diagnosis is in

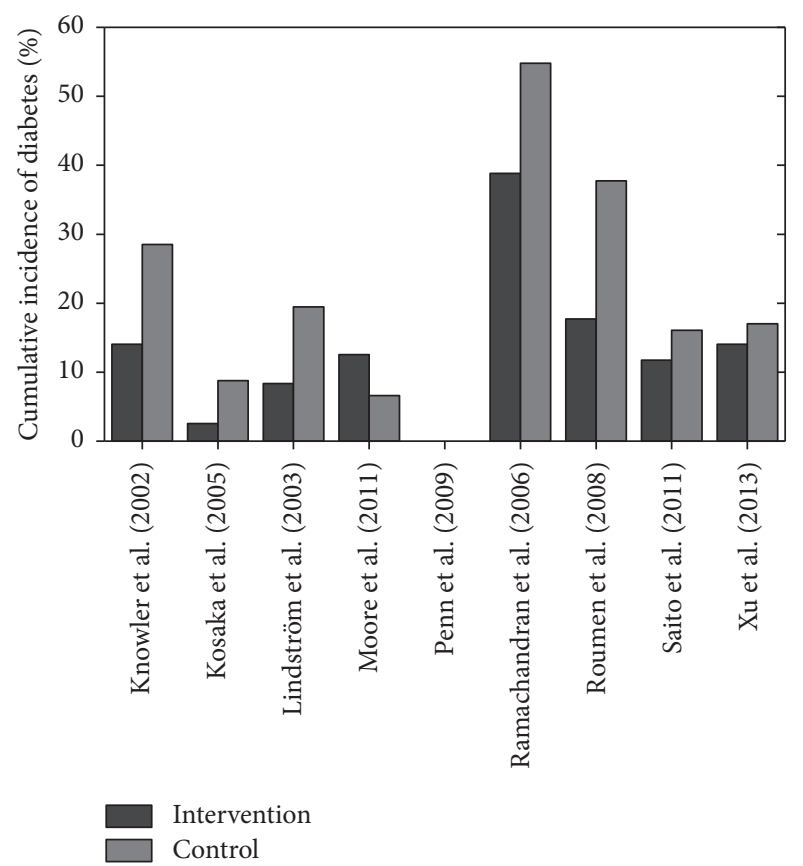

FIGURE 4: Cumulative incidence of diabetes for control and intervention groups.

accordance with the WHO [8] criteria for diagnosis, previously outlined in this review. Some of the studies had extra inclusion criteria which included age restrictions of participants $[17,19,21,24,25]$ and BMI above a certain threshold $[17,19,21,25]$.

Exclusion from all studies was due to diagnosis of diabetes before or during the study. Diagnosis of diabetes was completed by testing either fasting plasma glucose concentration or glucose concentration 2 hours after a 75 g oral glucose load. All studies, apart from Lindström et al.s [19] and Xu et al.'s [25] studies, state a numerical value for cut-off for diabetes diagnosis. This means that the reviewing author is unable to specify what method was used by Lindström et al. [19] and Xu et al. [25] to diagnose diabetes. Further exclusions for some studies were due to chronic illness that seriously reduced life expectancy or ability to partake in physical activity and medications that affect glucose concentration, mental illness, malignant neoplasm, and a range of kidney, liver, heart, and pancreas diseases.

\section{Characteristics of Interventions}

The characteristics of the study interventions are summarised in Table 3. All of the included studies promoted healthy eating and an increase in moderate physical activity. The delivery method varied in procedure and duration. The majority of studies used face-to-face individual interview style information, delivered by a dietician or a physiotherapist [17-19, 21, 23]. The remaining studies used either group based sessions carried out by a trained facilitator [20], utilised phone calls 


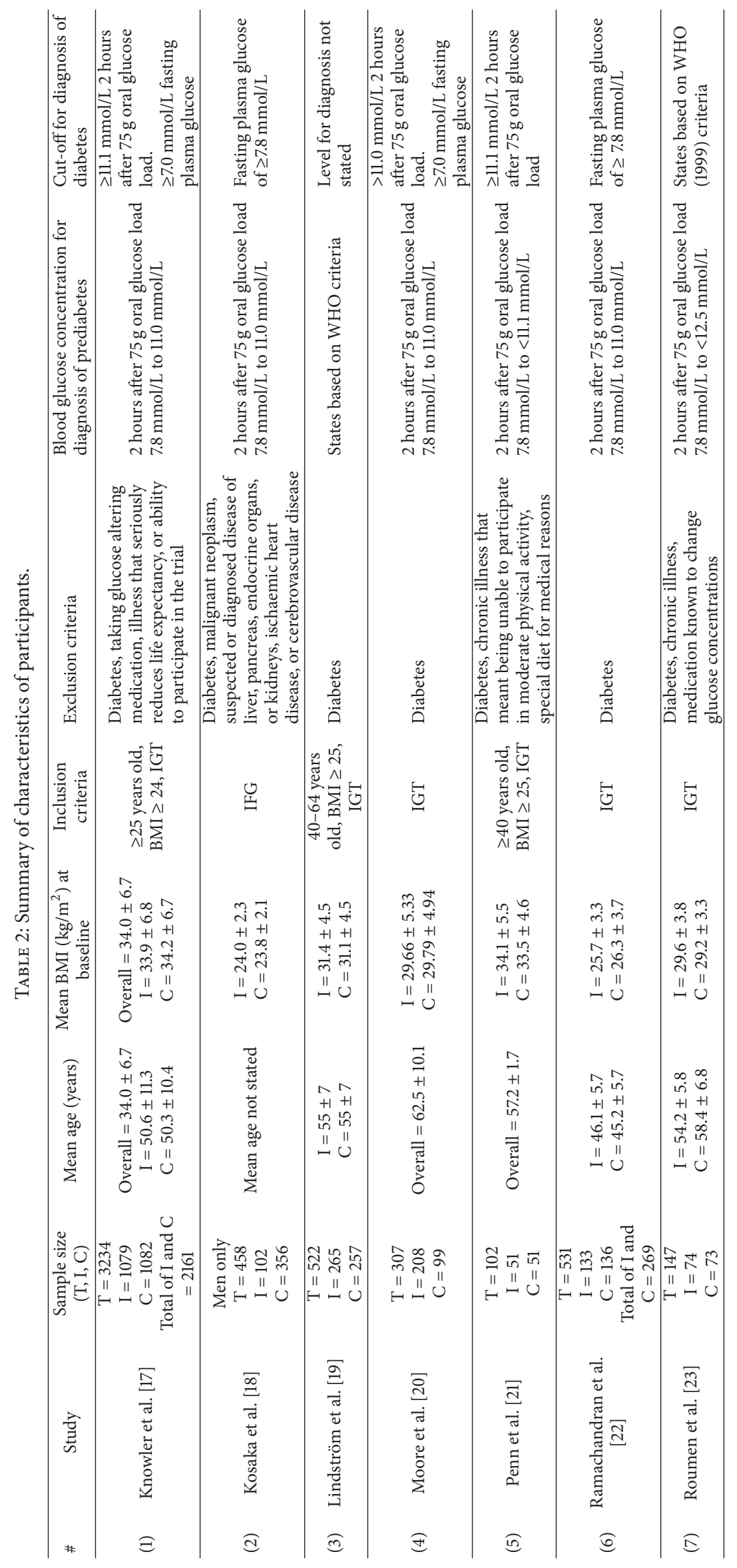




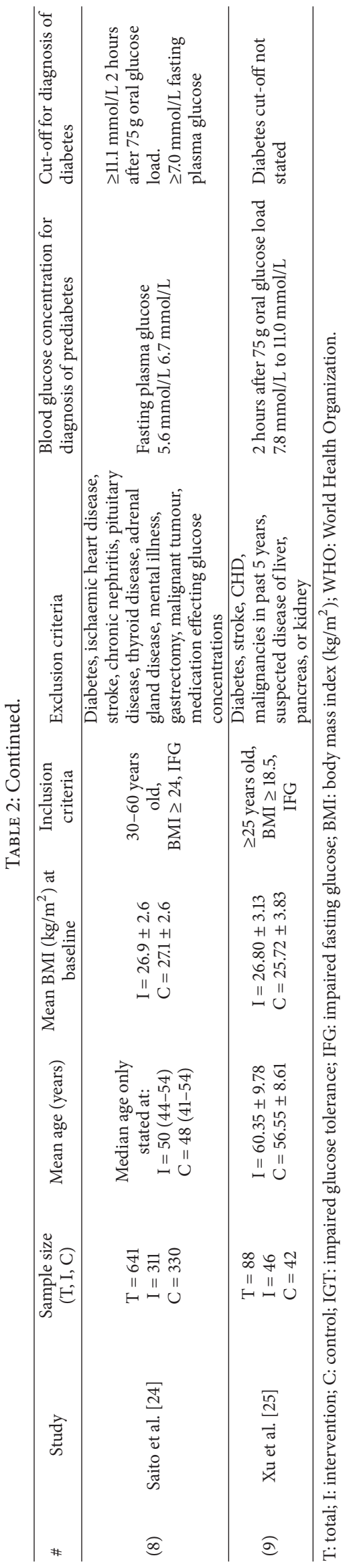




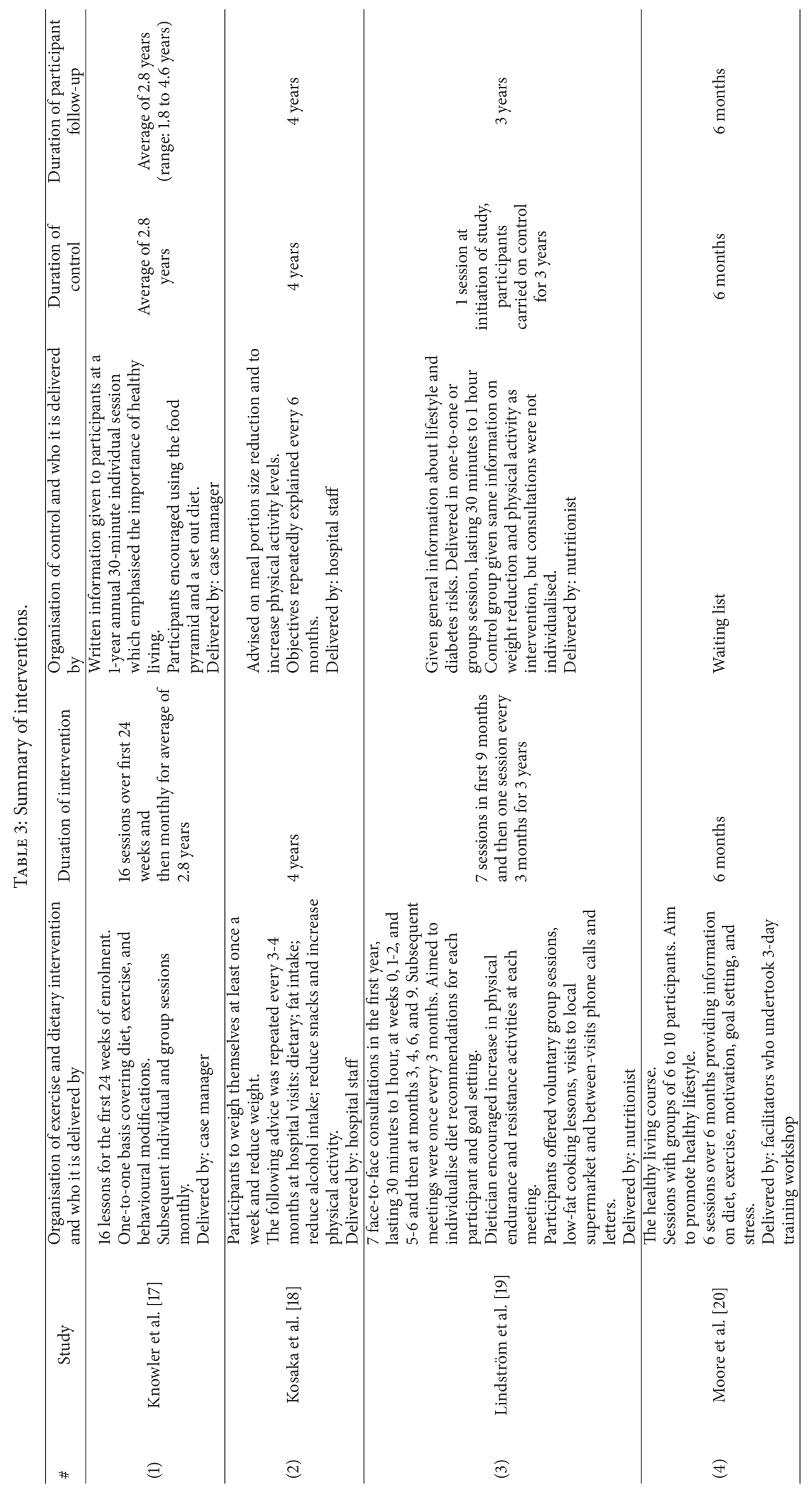




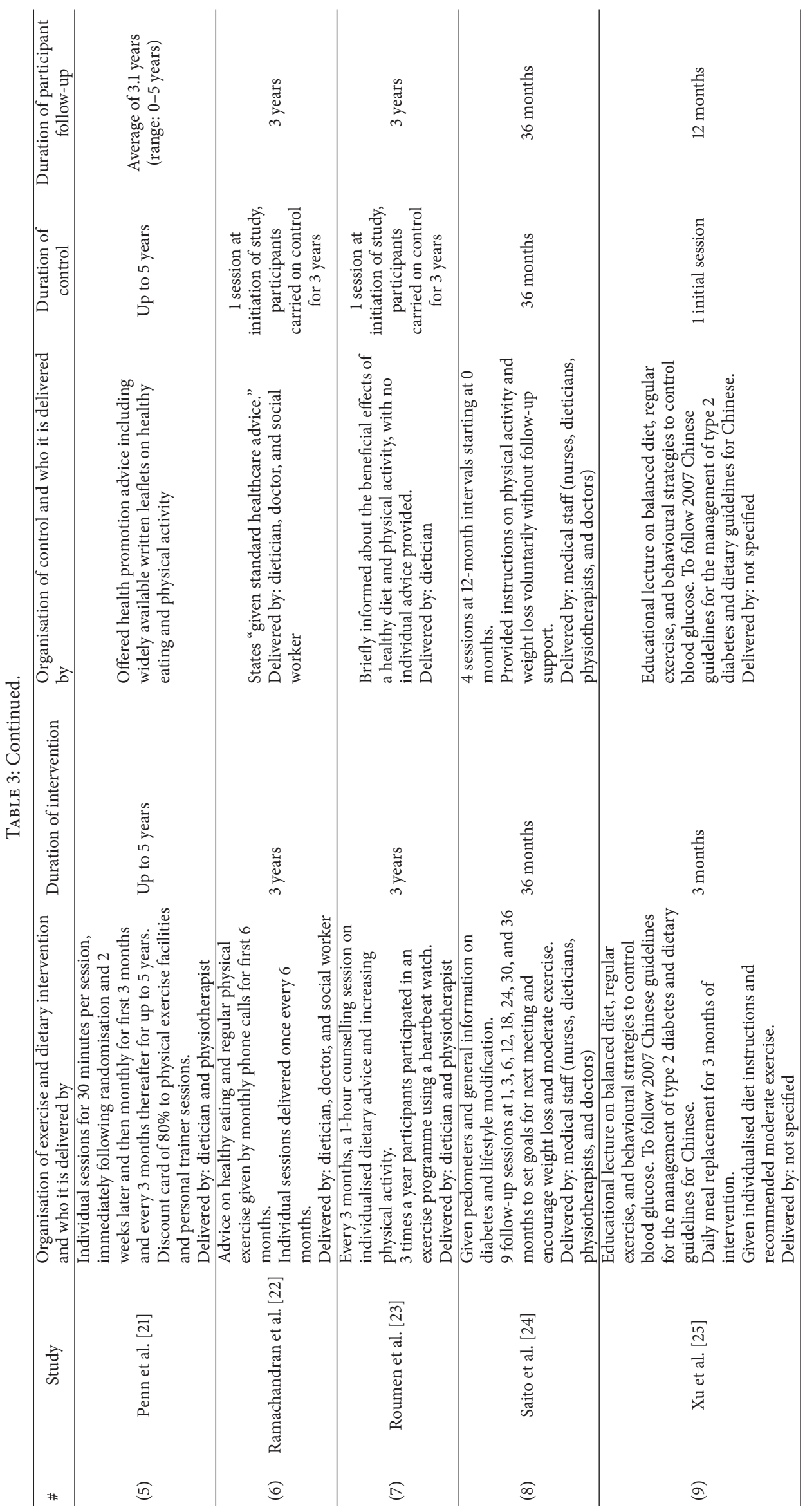


to provide information that coincided with 6 monthly faceto-face follow-up meetings [22], used a pedometer to selfmotivate participants to increase exercise with face-to-face meetings [24], or after initial educational lectures on diet and exercise were given 3 months of meal replacements [25]. The duration of intervention ranged from 6 months to 5 years; the majority of studies followed up participants for 36 months.

Three of the studies included offering further interventions. Lindström et al. [19] offered voluntary group sessions, low-fat cooking lessons, visits to local supermarkets, and between-visits phone calls and letters. Penn et al. [21] offered discount cards of $80 \%$ to physical exercise facilities and personal trainer sessions. Finally, Roumen et al. [23] had participants participate 3 times a year in an exercise programme using a heartbeat watch. Two of the studies included pharmacological interventions in separate arms [17, 22]; in these cases, and for the purposes of this review, the results of these arms are not reported.

All of the studies had weekly, monthly, or quarterly consultations with the participants to review blood glucose concentrations, weight, BMI, and food diaries among other individual study outcomes.

\section{Primary Outcome: Incidence of Diabetes}

The primary outcomes for this review are the incidence of diabetes development and glycaemic control. Table 4 summarises the primary outcome date. For each study, the baseline characteristics were assessed to have no significant differences between control and intervention groups which would affect the overall outcomes. Roumen et al. [23] had an age difference of 2 years between the control and intervention groups, the control group's mean age was 54 years and the intervention group's mean age was 52 years. The reviewing author judged this to be an insignificant difference.

Eight studies provided information for the cumulative incidence of diabetes for control and intervention groups. Penn et al. [21] provided information on the cumulative incidence difference between the intervention and control groups and not on each individual group. Figure 5 shows the cumulative incidence of diabetes breakdown for each study.

Cumulative incidence of diabetes is higher in the control group than in the intervention group in all cases, apart from Moore et al.s study [20]. The cumulative incidence for diabetes in the intervention and control groups, respectively, is as follows: Knowler et al. [17], 14.4\% and 28.9\%; Kosaka et al. [18], 3.0\% and 9.3\%; Lindström et al. [19], 9.0\% and 20.0\%; Moore et al. [20], 13.0\% and 7.0\%; Ramachandran et al. [22], $39.3 \%$ and 55\%; Roumen et al. [23], $18.0 \%$ and $38.0 \%$; Saito et al. [24], 12.2\% and 16.6\%; and $\mathrm{Xu}$ et al. [25], 14.6\% and $17.5 \%$. Penn et al. [21] had a cumulative incidence difference of diabetes diagnosis of $55 \%$ less in the intervention group compared to the control group.

Figure 5 shows the range of results for the intervention and control groups. The cumulative incidence of diabetes ranged from $3.0 \%$ [18] to $39.3 \%$ [22] for the intervention group, with a mean value of $15.44 \%$ (excluding [21]) across 8 studies. The cumulative incidence of diabetes for the control

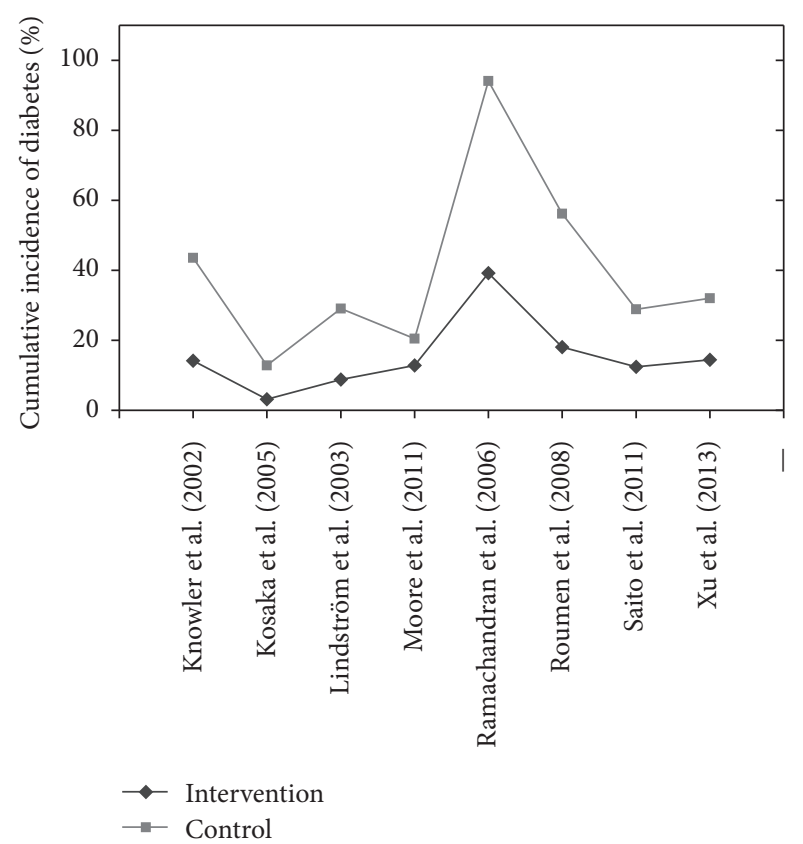

FIgURE 5: Cumulative incidence of diabetes across studies examined.

group ranged from $7.0 \%$ [20] to $38.0 \%$ [22], with a mean value of $24.01 \%$.

Two studies report incidence of diabetes in people-years. Knowler et al. [17] report that, in the intervention group, there were 4.8 cases per 100 people-years and, in the control group, there were 11.0 cases per 100 people-years. Penn et al. [21] had 32.7 cases per 1000 people-years for the intervention group and 67.1 cases per 1000 people-years for the control group.

\section{Primary Outcome: Glycaemic Control}

Four studies [17, 21, 22, 24] do not use glycaemic control as either a primary or a secondary outcome. The remaining studies reported glycaemic control in a number of ways, ranging from improvement in oral glucose tolerance test (OGTT) [18] to glycaemic values staying within prediabetes range [20], change from prediabetes to normoglycaemia [20, 25], and 2-hour plasma glucose levels to show positive or negative change from baseline $[19,20,23,25]$.

Kosaka et al. [18] reported OGTT improvement from baseline to end of study at 4 years. Improvement of $53.8 \%$ and $33.9 \%$ was observed for the intervention group and the control group, respectively. Moore et al. [20] reported cumulative incidence of prediabetes of $45 \%$ for intervention group and $67 \%$ for control group at the end of the study. Two studies reported change from prediabetes to normoglycaemia. Moore et al. [20] had results of $43 \%$ for the intervention group and $26 \%$ for control group. Xu et al. [25] reported results of $39 \%$ for intervention group and $7.5 \%$ for control group.

The four studies that reported 2-hour plasma glucose levels represented data by showing actual glycaemic levels of difference in glycaemic levels from baseline to the end of 


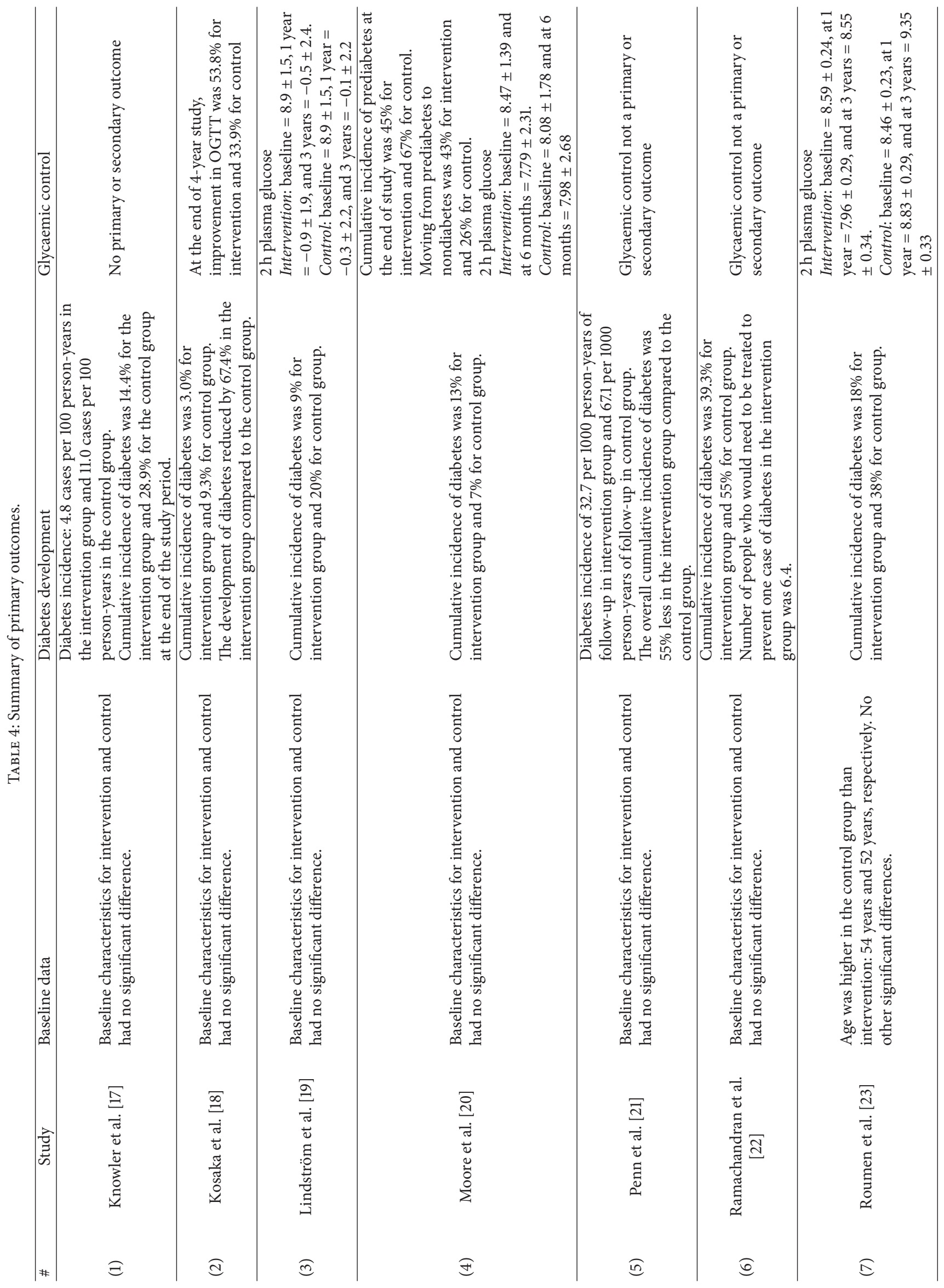




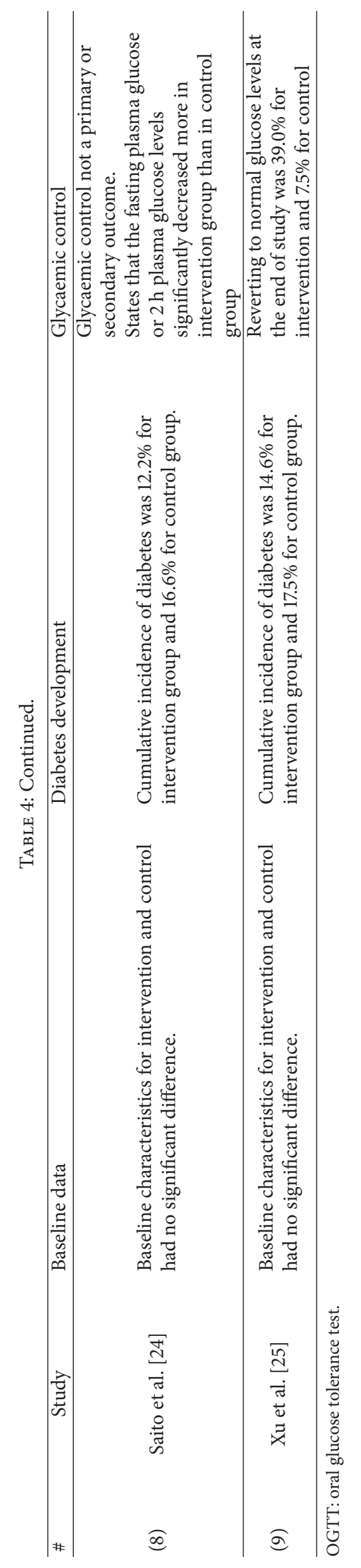


the study. Two studies showing actual glycaemic levels had results. Roumen et al. [23] had intervention group's results as follows: baseline, $8.59 \pm 0.24,1$ year, $7.96 \pm 0.29$, and 3 years, $8.55 \pm 0.32$; and they had control group's results as follows: baseline, $8.46 \pm 0.23,1$ year, $8.83 \pm 0.29$, and 3 years, $9.35 \pm 0.33$. Moore et al. [20] had intervention group's results as follows: baseline, $8.47 \pm 1.39$, and 6 months, $7.79 \pm$ 2.31; and they had control group's results as follows: baseline, $8.08 \pm 1.78$, and at 6 months, $7.98 \pm 2.68$. Two studies used difference in glycaemic control levels. Xu et al. [25] had intervention group's results as follows: baseline, $8.90 \pm 1.25$, with change at 1 year of $-1.24 \pm 0.35$; and they had control group's results as follows: baseline, $9.24 \pm 1.58$, with change at 1 year of $+0.85 \pm 0.86$. Lindström et al. [19] had intervention group's results as follows: baseline, $8.9 \pm 1.5$, change at 1 year, $-0.9 \pm 1.9$, and change at 3 years, $-0.5 \pm 2.4$; and they had control group's results as follows: baseline, $8.9 \pm 1.5$, change at 1 year, $-0.3 \pm 2.2$, and change at 3 years, $-0.1 \pm 2.2$. For the studies that reported glycaemic control, the intervention groups had vastly more improvements in 2-hour plasma glucose concentration, glycaemic levels reverting to normal, and less participants remaining with prediabetes compared to the control groups.

\section{Secondary Outcome: Physical Exercise Capacity}

The results of the secondary outcomes are summarised in Table 5. Four of the 9 studies reported on physical exercise capacity by meeting recommended goals, maximum oxygen volume $\left(\mathrm{VO}_{2}\right)$, or leisure time and other time physical activities. The 5 other studies either did not report physical activity levels or did not report sufficient details about them to be included in the results.

Knowler et al. [17] reported how many intervention group participants were meeting 150 minutes of physical activity a week. The study had intense input up to the 24 th week mark. At this point, $74 \%$ of participants were completing 150 minutes of physical activity a week. The level of adherence dropped to $58 \%$ by the end of the study. Levels for the control group were not reported.

Lindström et al. [19] reported that moderate to vigorous leisure time activity increases more significantly in the intervention group compared to the control group. Results were reported in minutes per week of exercise. The control group at baseline was achieving 21 minutes, increasing marginally to 23 minutes at the end of the study. Intervention group's baseline results were 16 minutes, increasing to 50 minutes per week by the end of the study.

Ramachandran et al. [22] did not report specific figures for physical exercise adherence; a graph is provided but it is difficult to ascertain specific values from this. The study does state that adherence levels changed from $41.7 \%$ at baseline to $58.8 \%$ at the end of the study for the intervention group.

Roumen et al. [23] reported both $\mathrm{VO}_{2}$ (ltr/min) max and days where 30 minutes of activity was met. The $\mathrm{VO}_{2}$ level for the intervention group at baseline was $2.22 \pm 0.61$, changing at 1 year by $+0.13 \pm 0.25$, at 2 years by $+0.10 \pm 0.25$, and at 3 years by $+0.05 \pm 0.35$. The control group's $\max \mathrm{VO}_{2}$ at baseline was $2.13 \pm 0.55$, changing at 1 year by $+0.02 \pm 0.21$, at 2 years by $-0.05 \pm 0.23$, and at 3 years by $-0.06 \pm 0.21$. The number of days the target of 30-minute activity was being met changes for the intervention group by $+0.89 \pm 2.75$ days and for the control group by $-0.55 \pm 3.31$ days.

\section{Secondary Outcome: Change in BMI}

Four studies reported change in BMI. Moore et al. [20] reported BMI change for the intervention group from 29.66 \pm 5.33 at baseline to $28.72 \pm 5.00$ at 6 months. The change for the control group was from $29.76 \pm 4.94$ at baseline to $29.50 \pm$ 5.24 at 6 months. These values indicate decreases of 0.94 for the intervention group and 0.29 for the control group.

The 3 remaining studies reported difference in BMI from baseline at different stages of the studies. Lindström et al. [19] had baseline results of $31.4 \pm 4.5$ for the intervention group, changing at 1 year by $-1.6 \pm 1.8$ and at 3 years by $-1.3 \pm 1.9$. They had baseline results of $31.1 \pm 4.5$ for the control group, changing at 1 year by $-0.4 \pm 1.3$ and at 3 years by $-0.3 \pm 2.0$. Roumen et al. [23] reported baseline results of $29.6 \pm 3.8$ for the intervention group, changing at 1 year by $-0.94 \pm 1.25$, at 2 years by $-0.61 \pm 1.49$, and at 3 years by $-0.36 \pm 1.47$. They had baseline results of $29.2 \pm 3.3$ for the control group, changing at 1 year by $-0.20 \pm 1.39$, at 2 years by $-0.02 \pm 1.17$, and at 3 years by $+0.08 \pm 1.80$. Finally, Xu et al. [25] had baseline results of $26.80 \pm 3.13$ for the intervention group, changing at 1 year by $-0.66 \pm 0.13$. They had baseline results of $25.72 \pm 3.83$ for the control group, changing at 1 year by $-0.22 \pm 0.15$.

\section{Secondary Outcome: Change in Weight}

All of the studies reported on changes in mean weight $(\mathrm{kg})$ lost over the study periods. Ramachandran et al. [22] states no significant weight change for intervention group. The 8 remaining studies either provided the baseline and end-ofstudy mean weights or provided the difference observed from baseline to the end of the study.

Moore et al. [20] states that the baseline data for the intervention group was $80.7 \pm 16.01$ and at the end of the study it was $78.11 \pm 14.98$. The control group had baseline data of $82.02 \pm 16.27$ and at the end of the study it was $81.20 \pm 17.39$.

Four studies reported mean weight change from baseline to the end of the study. Knowler et al. (2008) had a mean weight difference of $-5.6 \mathrm{~kg}$ for the intervention group and $-0.1 \mathrm{~kg}$ for the control group. Kosaka et al. [18] had mean weight change of $-2.18 \mathrm{~kg}$ for the intervention group and $-0.39 \mathrm{~kg}$ for the control group. Penn et al. [21] had change of $-2.3 \mathrm{~kg}$ for intervention group and change of $+0.01 \mathrm{~kg}$ for the control group. Finally, Saito et al. [24] had weight change of $-2.5 \mathrm{~kg}$ and $-1.1 \mathrm{~kg}$ for the intervention group and the control group, respectively.

The remaining 3 studies reported their findings in more detail. Lindström et al. [19] had baseline results of $86.7 \pm 14.0$ for intervention group, changing after 1 year by $-4.5 \pm 5.0$ and at 3 years by $-3.5 \pm 5.1$. They had baseline results of $85.5 \pm$ 14.4 for control group, changing after 1 year by $-1.0 \pm 3.7$ and 


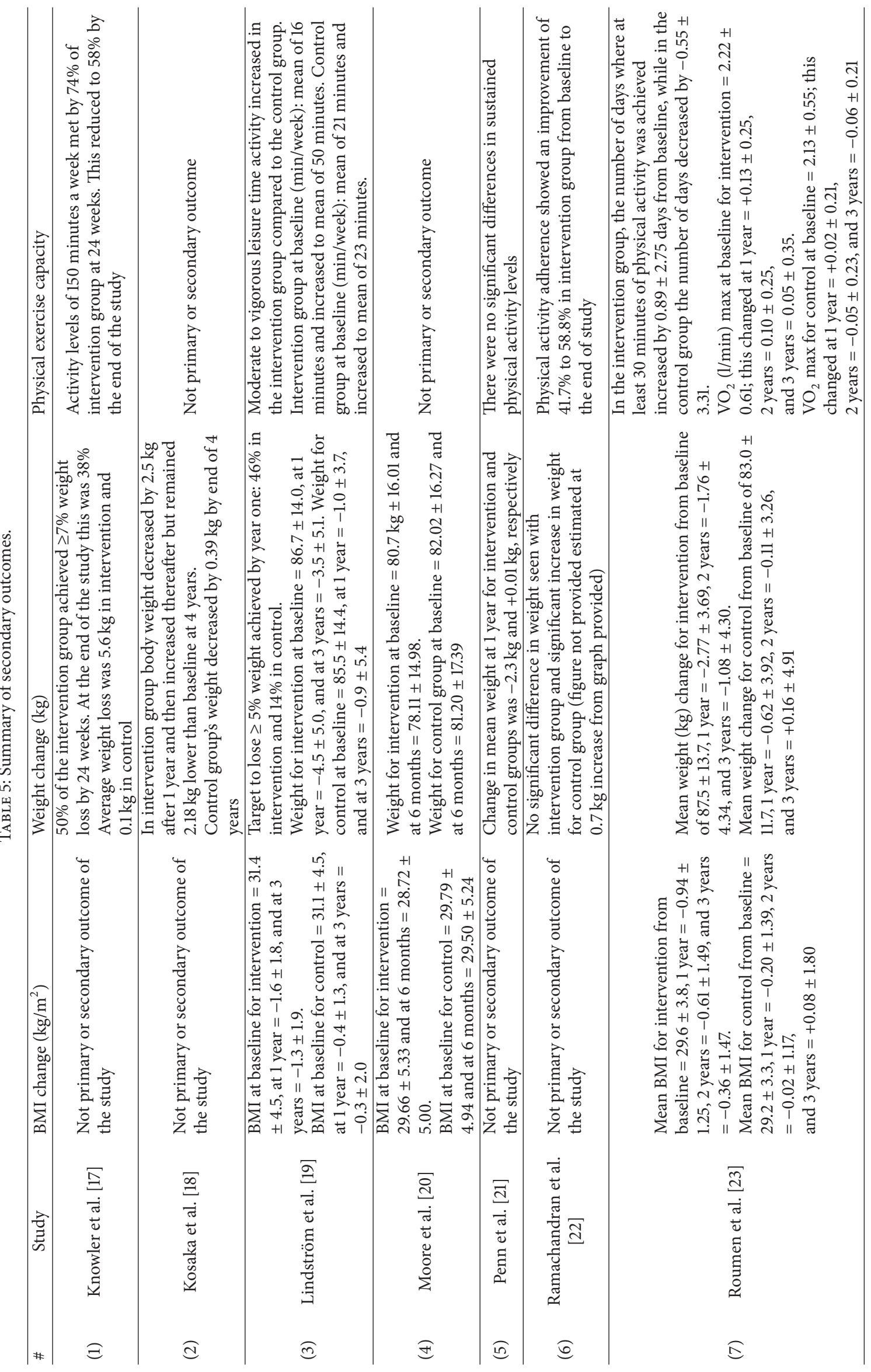







at 3 years by $-0.9 \pm 5.4$. Roumen et al. [23] reported baseline results of $87.5 \pm 13.7$ for intervention group, changing at 1 year by $-2.77 \pm 3.69$, at 2 years by $-1.76 \pm 4.34$, and at 3 years by $-1.08 \pm 4.30$. They had baseline results of $83.0 \pm 11.7$ for control group, changing at 1 year by $-0.62 \pm 3.92$, at 2 years by $-0.22 \pm 3.26$, and at 3 years by $+0.16 \pm 4.91$. Finally, Xu et al. [25] had baseline results of $68.24 \pm 9.73$ for intervention group, changing at 1 year by $-1.75 \pm 0.35$. They had baseline results of $69.69 \pm 1.36$ for control group, changing at 1 year by $-0.55 \pm 0.40$.

Three of the studies further reported how many participants met the weight loss goal target. Knowler et al. (2008) had a weight loss target of $\geq 7 \%$; at 24 weeks (end of intense input) $50 \%$ of the intervention group had achieved this. This figure dropped at the end of the study to $38 \%$ of participants. Lindström et al. [19] had target weight loss of $\geq 5 \%$. At 1 year, $46 \%$ of the intervention group and $14 \%$ of the control group had achieved this target. Saito et al. [24] had target weight loss of $\geq 5 \%$; $32 \%$ of the intervention group and $18 \%$ of the control group achieved this level.

\section{Discussion}

12.1. Incidence of Diabetes. The results advocate lifestyle intervention to be utilised for effectively delaying or preventing the progression of prediabetes to T2DM. All of the studies, besides Moore et al.s study [20], had a reduced incidence of diabetes with lifestyle intervention being adopted in comparison to the control (normal treatment). These findings correlate with previous studies assessing whether lifestyle interventions could conceivably be adopted to reduce the incidence of diabetes [13, 15, 26-28].

De Vegt et al. [29] found that, over a span of 6.4 years, $34 \%$ of their study subjects, with baseline IGT, naturally developed T2DM. Similarly, Meigs et al. [30] found that 21\% of their study subjects, with baseline IFG or IGT, progressed naturally to T2DM during a 5-year study. Seven of the 9 studies had diabetes incidence rates for the intervention groups below Meigs et al.s [30] and De Vegt et al.s [29] findings. Determining lifestyle intervention reduced the likelihood of T2DM diagnosis more than natural progression. Two studies were above the natural progression threshold [21, 22]. Penn et al. [21] did not report cumulative incidence of diabetes, so comparison was unavailable. Ramachandran et al. [22] had an intervention group's cumulative incidence rate for diabetes of $39.3 \%$; this was a spike when compared to other studies (Figures 4 and 5). However, study participants were native Asian Indians who are physiologically more susceptible to diabetes due to their ethnicity [31, 32]. The natural progression studies were not completed on such high risk groups. Furthermore, Ramachandran et al.s [22] control group had diabetes incidence rate of 55\%, showing that lifestyle intervention reduced the incidence of diabetes compared to the control.

Moore et al's study [20] was the only one whose control group had a lower incidence of diabetes than the intervention. This was also the only study to use group based sessions to instruct the intervention group. This tentatively indicates that group based approach may not be as effective as oneto-one sessions for lifestyle advice. Interventions need to be individualised and performed by a skilled healthcare professional trained in the specific field. The results obtained in this study may be due Moore et al. focusing on reducing diabetes. However, incidence of diabetes reduction for the intervention group was similar to the other included studies. Furthermore, follow-up was only for 6 months; longer followup might affect the results.

12.2. Glycaemic Control. Five studies reported glycaemic control as a primary or secondary outcome. Results revealed that a considerable proportion of participants in the intervention groups reverted to normoglycaemia at an increased rate compared to the control group. Furthermore, significant reductions in 2-hour plasma glucose concentrations were present with lifestyle intervention in the short term. Likewise, there was a reduction in 2-hour plasma glucose levels for the control groups; however, levels are substantially less significant.

Results indicated obstacles associated with long-term maintenance of improved glycaemic regulation. Two studies reported glycaemic control at 3 points $[19,23]$ : baseline, 1 year, and 3 years. For both studies, mean glycaemic concentration increased at year 3 compared to year 1 after the initial drop from baseline observed at year 1 . These findings were from two studies only, so they should be interpreted with caution. Overall, results suggested that lifestyle intervention in the short term had an undeniable positive effect for glycaemic control but long-term maintenance problems. Different individuals require different motivational input to achieve behavioural changes; this could range from identification of prediabetes at baseline to continuous support to change behaviours. Findings by Norris et al. [33] and Yoon et al. [34] correlate with this review's findings, supporting the effectiveness of lifestyle adaptation in the short term but with long-term adherence complications.

All included studies had intensive initial period for study intervention. After this, face-to-face contact reduced in frequency. Improvements in short-term glycaemic control may be associated with the initial intense intervention. When intensity dropped, so did glycaemic control. Kim and $\mathrm{Oh}$ [35] found that an intervention group with intense healthcare input, via regular phone call contact, had better lifestyle adherence and consequently improved glycaemic control than a control group with standard healthcare input. These findings correlate with those of the review. However, in contrast, Radhakrishnan [36] found that standard care may be as effective as tailored individualised care for selfmanagement behaviours in long-term conditions. The motivational effect of intense input needs to be assessed separately and considered in future RCTs.

Improvements in glycaemic control could have been attributed to some of the study subjects volunteering and already having willingness to change [37]. This is likely to lead to subsequent changes in lifestyle, which will benefit the individual's glycaemic control. For lifestyle interventions to work, individuals need the initial willingness or intention to change so they are prepared to persevere with lifestyle modifications over the long term [38]. 
12.3. Physical Exercise Capacity. Physical exercise capacity was reported in 4 studies. Improvements in exercise capacity for the intervention groups were more significant than for the control group. Although increases in physical exercise capacity were observed, the levels remain low for participants achieving 150 minutes of activity a week. Herbst et al. [39] found in adolescents with T2DM that over half of the participants did not perform regular physical activity. This is not just the case for T2DM; it is apparent with other long-term conditions. Serour and Alqhenaei [40] found that individuals at risk of cardiovascular disease did not complete exercise programmes, even though they were fully aware of the positive effects exercise has on their condition.

Physical exercise capacity at the end of the intense intervention period compared with the end of the trial deteriorated; however, it improved from baseline. There was high exercise capacity in the short term, which reduced as intervention intensity did. This was also found to be the case in Woodard and Berry [41] and Madden et al.'s [42] works. Furthermore, Tran et al. [43] found that, after the diabetes aerobic and resistance exercise (DARE) trial, only $41 \%$ of participants completed regular exercise 8 to 12 years after an intensive 6-month trial. A positive correlation exists between glycaemic control and physical exercise capacity. Exercise only trials are needed to assess how exercise affects glycaemic control.

Exercise and motivational problems were observed. Tulloch et al. (2013) found that continuous intervention with exercise specialists is needed to maintain exercise levels. Furthermore, Visram et al. [44] found that newly diagnosed diabetics had fears and lack of understanding about exercise which acted as a barrier. In terms of the practical implications of this study, the assessment of self-efficacy in people with prediabetes may need to be the first step in the development of individualised lifestyle interventions and additional booster sessions to improve long-term exercise capacity [46].

12.4. Weight and BMI. In 8 studies, lifestyle intervention significantly reduced BMI and weight, implying that lifestyle intervention involving diet and exercise is an effective treatment for weight reduction for adults with prediabetes. These results are not surprising as lifestyle adaptation has previously been recognised as effective for weight and BMI reduction with improvements in glycaemic control also identified [47, 48].

The same pattern that was present with exercise capacity and glycaemic control is evident with weight and BMI change. Over the short term, there was significant improvement in weight. However, over the long term, both weight and BMI increased. The results are in line with those of Norris et al. [49] and Kouvelioti et al. [50] in two separate systematic reviews of weight loss.

Weight and BMI regain occurred in the intervention group but levels still remained significantly below the baseline results, whereas, for some cases in the control groups, weight regain above the original baseline results was observed. Even with modest weight loss, every kilogram of weight loss is associated with a $16 \%$ reduction in diabetes risk [51] and has long-lasting effects for T2DM risk reduction [52]. The results from the review suggest that small percentages of sustained weight change are beneficial in reducing the risk of prediabetes progression to T2DM and improving glycaemic control.

\section{Conclusion}

This systematic review aimed to assess the feasibility of lifestyle interventions being used to treat prediabetes and enhance glycaemic control. The outcome measures were to assess cumulative incidence of diabetes development, glycaemic control, physical exercise capacity, and changes in weight/BMI. Nine RCTs met the inclusion criteria from a total of 1,784 relevant studies searched. The review provides evidence for the effectiveness of lifestyle interventions to treat prediabetes. Overall, cumulative incidence of diabetes is drastically reduced in the intervention groups compared to control groups (standard care). Furthermore, glycaemic control was improved in the short term, with many participants reverting to normoglycaemia. In the long term, glycaemic control diminished, but glycaemic control was still superiorly better managed than baseline results and control groups. A similar scenario is true for weight and BMI, where short-term reductions are replaced by long-term weight and BMI increases. Physical exercise capacity improved at an increased rate in the intervention groups compared to the control groups; however, it is still significantly lower than the recommended 150 minutes of exercise a week.

As a result of the findings from this review, lifestyle intervention should be provided as a treatment option for adult prediabetes patients to improve glycaemic control and reduce the prospect of their condition developing into type 2 diabetes mellitus. However, before this is implemented in a practice setting, more research needs to be completed to assess how motivation to change can be maintained over the long term. Furthermore, RCTs with large participant numbers, completed in the UK, need to be undertaken to assess the generalizability of lifestyle intervention treatments for patients accessing care from the NHS.

\section{Conflicts of Interest}

The authors declare that there are no conflicts of interest regarding the publication of this paper.

\section{Acknowledgments}

The authors would like to thank the Independent Diabetes Trust (IDDT) for funding.

\section{References}

[1] L. Guariguata, D. R. Whiting, I. Hambleton, J. Beagley, U. Linnenkamp, and J. E. Shaw, "Global estimates of diabetes prevalence for 2013 and projections for 2035," Diabetes Research and Clinical Practice, vol. 103, no. 2, pp. 137-149, 2014.

[2] D. Meetoo, "Diabetes: complications and the economic burden," British Journal of Health Care Management, vol. 20, no. 2, pp. 60-67, 2014. 
[3] Health and Social Care Information Centre (HSCIC), Quality and Outcomes Framework Achievement, Prevalence and Exception Data, 2012/13, 2013, http://www.hscic.gov.uk/catalogue/ PUB12262/qual-outc-fram-12-13-rep.pdf.

[4] N. Hex, C. Bartlett, D. Wright, M. Taylor, and D. Varley, "Estimating the current and future costs of Typel and Type2 diabetes in the UK, including direct health costs and indirect societal and productivity costs," Diabetic Medicine, vol. 29, no. 7, pp. 855-862, 2012.

[5] Department of Health (DoH), Turning the Corner: Improving Diabetes Care, 2006, http://www.bipsolutions.com/docstore/ pdf/13587.pdf.

[6] National Institute for Health and Care Excellence (NICE), Preventing Type 2 Diabetes: Risk Identification and Intervention for Individuals at High Risk, 2012, http://www.nice.org.uk/ guidance/ph38/chapter/1-recommendations.

[7] American Diabetes Association, "Diagnosis and classification of diabetes mellitus," Diabetes Care, vol. 28, supplement 1, pp. S37-S42, 2004.

[8] World Health Organization (WHO), "Definition and diagnosis of diabetes mellitus and intermediate hyperglycaemia," 2006, http://whqlibdoc.who.int/publications/2006/9241594934_eng .pdf?ua $=1$.

[9] S. Genuth, K. G. M. M. Alberti, P. Bennett et al., "Follow-up report on the diagnosis of diabetes mellitus," Diabetes Care, vol. 26, no. 11, pp. 3160-3167, 2003.

[10] M. Bergman, "Inadequacies of absolute threshold levels for diagnosing prediabetes," Diabetes/Metabolism Research and Reviews, vol. 26, no. 1, pp. 3-6, 2010.

[11] M. B. Davidson, P. B. Landsman, and C. M. Alexander, "Lowering the criterion for impaired fasting glucose will not provide clinical benefit," Diabetes Care, vol. 26, no. 12, pp. 3329-3330, 2003.

[12] International Expert Committee, "International Expert Committee Report on the role of the A1C assay in the diagnosis of diabetes," Diabetes Care, vol. 32, no. 7, pp. 1327-1334, 2009.

[13] X.-R. Pan, G.-W. Li, Y.-H. Hu et al., "Effects of diet and exercise in preventing NIDDM in people with impaired glucose tolerance: The Da Qing IGT and diabetes study," Diabetes Care, vol. 20, no. 4, pp. 537-544, 1997.

[14] J. C. Oldroyd, N. C. Unwin, M. White, J. C. Mathers, and K. G. M. M. Alberti, "Randomised controlled trial evaluating lifestyle interventions in people with impaired glucose tolerance," Diabetes Research and Clinical Practice, vol. 72, no. 2, pp. 117-127, 2006.

[15] L. Orozco, A. Buchleitner, G. Gimenez-Perez, M. Figuls, B. Richter, and D. Mauricio, "Exercise or exercise and diet for preventing type 2 diabetes mellitus [systematic review]," The Cochrane Database of Systematic Reviews, vol. 3, Article ID CD003054, Chichester, UK: John Wiley and Sons, Ltd, 2008.

[16] B. Lindahl, T. K. Nilssön, K. Borch-Johnsen et al., "A randomized lifestyle intervention with 5-year follow-up in subjects with impaired glucose tolerance: pronounced short-term impact but long-term adherence problems," Scandinavian Journal of Public Health, vol. 37, no. 4, pp. 434-442, 2009.

[17] W. C. Knowler, E. Barrett-Connor, S. E. Fowler et al., "Reduction in the incidence of type 2 diabetes with lifestyle intervention or metformin," New England Journal of Medicine, vol. 346, no. 6, pp. 393-403, 2002.

[18] K. Kosaka, M. Noda, and T. Kuzuya, "Prevention of type 2 diabetes by lifestyle intervention: a Japanese trial in IGT males,"
Diabetes Research and Clinical Practice, vol. 67, no. 2, pp. 152 162, 2005.

[19] J. Lindström, A. Louheranta, M. Mannelin et al., "The Finnish Diabetes Prevention Study (DPS): lifestyle intervention and 3year results on diet and physical activity," Diabetes Care, vol. 26, no. 12, pp. 3230-3236, 2003.

[20] S. M. Moore, E. A. Hardie, N. J. Hackworth et al., "Can the onset of type 2 diabetes be delayed by a group-based lifestyle intervention? A randomised control trial," Psychology and Health, vol. 26, no. 4, pp. 485-499, 2011.

[21] L. Penn, M. White, J. Oldroyd, M. Walker, K. G. M. M. Alberti, and J. C. Mathers, "Prevention of type 2 diabetes in adults with impaired glucose tolerance: The European Diabetes Prevention RCT in Newcastle upon Tyne, UK," BMC Public Health, vol. 9, no. 1, article 342, 2009.

[22] A. Ramachandran, C. Snehalatha, S. Mary, B. Mukesh, A. D. Bhaskar, and V. Vijay, "The Indian Diabetes Prevention Programme shows that lifestyle modification and metformin prevent type 2 diabetes in Asian Indian subjects with impaired glucose tolerance (IDPP-1)," Diabetologia, vol. 49, no. 2, pp. 289297, 2006.

[23] C. Roumen, E. Corpeleijn, E. J. M. Feskens, M. Mensink, W. H. M. Saris, and E. E. Blaak, "Impact of 3-year lifestyle intervention on postprandial glucose metabolism: the SLIM study," Diabetic Medicine, vol. 25, no. 5, pp. 597-605, 2008.

[24] T. Saito, M. Watanabe, J. Nishida et al., "Lifestyle modification and prevention of type 2 diabetes in overweight Japanese with impaired fasting glucose levels: a randomized controlled trial," Archives of Internal Medicine, vol. 171, no. 15, pp. 1352-1360, 2011.

[25] Y. Xu, L. Wang, J. He et al., "Prevalence and control of diabetes in Chinese adults," The Journal of the American Medical Association, vol. 310, no. 9, pp. 948-958, 2013.

[26] J. Tuomilehto, J. Lindström, J. G. Eriksson et al., "Prevention of type 2 diabetes mellitus by changes in lifestyle among subjects with impaired glucose tolerance," The New England Journal of Medicine, vol. 344, no. 18, pp. 1343-1350, 2001.

[27] C. L. Gillies, K. R. Abrams, P. C. Lambert et al., "Pharmacological and lifestyle interventions to prevent or delay type 2 diabetes in people with impaired glucose tolerance: systematic review and meta-analysis," British Medical Journal, vol. 334, no. 7588, pp. 299-302, 2007.

[28] C. Y. Jeon, R. P. Lokken, F. B. Hu, and R. M. Van Dam, "Physical activity of moderate intensity and risk of type 2 diabetes: a systematic review," Diabetes Care, vol. 30, no. 3, pp. 744-752, 2007.

[29] F. De Vegt, J. M. Dekker, A. Jager et al., "Relation of impaired fasting and postload glucose with incident type 2 diabetes in a Dutch population: the Hoorn study," The Journal of the American Medical Association, vol. 285, no. 16, pp. 2109-2113, 2001.

[30] J. B. Meigs, D. C. Muller, D. M. Nathan, D. R. Blake, and R. Andres, "The natural history of progression from normal glucose tolerance to type 2 diabetes in the Baltimore Longitudinal Study of Aging," Diabetes, vol. 52, no. 6, pp. 1475-1484, 2003.

[31] D. W. Lam and D. LeRoith, "The worldwide diabetes epidemic," Current Opinion in Endocrinology, Diabetes and Obesity, vol. 19, no. 2, pp. 93-96, 2012.

[32] A. Ramachandran, R. C. Wan Ma, and C. Snehalatha, "Diabetes in Asia," The Lancet, vol. 375, no. 9712, pp. 408-418, 2010.

[33] S. L. Norris, M. M. Engelgau, and K. M. V. Narayan, "Effectiveness of self-management training in type 2 diabetes: a 
systematic review of randomized controlled trials," Diabetes Care, vol. 24, no. 3, pp. 561-587, 2001.

[34] U. Yoon, L. L. Kwok, and A. Magkidis, "Efficacy of lifestyle interventions in reducing diabetes incidence in patients with impaired glucose tolerance: a systematic review of randomized controlled trials," Metabolism: Clinical and Experimental, vol. 62, no. 2, pp. 303-314, 2013.

[35] H.-S. Kim and J.-A. Oh, "Adherence to diabetes control recommendations: impact of nurse telephone calls," Journal of Advanced Nursing, vol. 44, no. 3, pp. 256-261, 2003.

[36] K. Radhakrishnan, "The efficacy of tailored interventions for self-management outcomes of type 2 diabetes, hypertension or heart disease: a systematic review," Journal of Advanced Nursing, vol. 68, no. 3, pp. 496-510, 2012.

[37] G. Heiman, Research Methods in Psychology, Houghton Mifflin Harcourt, Boston, Mass, USA, 3rd edition, 2002.

[38] V. Heiss and R. Petosa, "Correlates of physical activity among adults with type 2 diabetes: a systematic literature review," American Journal of Health Education, vol. 45, no. 5, pp. 278287, 2014.

[39] A. Herbst, T. Kapellen, E. Schober, C. Graf, T. Meissner, and R. Holl, "Impact of regular physical activity on blood glucose control and cardiovascular risk factors in adolescents with type 2 diabetes mellitus-a multicenter study of 578 patients from 225 centres," Pediatric Diabetes, vol. 16, no. 3, pp. 204-210, 2015.

[40] M. Serour and H. Alqhenaei, "Cultural factors and patients adherence to lifestyle measures," The British Journal of General Practice, vol. 57, no. 537, pp. 291-295, 2007.

[41] C. M. Woodard and M. J. Berry, "Enhancing adherence to prescribed exercise: structured behavioral interventions in clinical exercise programs," Journal of Cardiopulmonary Rehabilitation, vol. 21, no. 4, pp. 201-209, 2001.

[42] S. G. Madden, S. J. Loeb, and C. A. Smith, "An integrative literature review of lifestyle interventions for the prevention of type II diabetes mellitus," Journal of Clinical Nursing, vol. 17, no. 17, pp. 2243-2256, 2008.

[43] C. S. Tran, Y. Kong, A. Gupta et al., "Long-term exercise adherence after completion of the Diabetes Aerobic and Resistance Exercise (DARE) trial," Canadian Journal of Diabetes, vol. 37, supplement 4, pp. S50-S51, 2013.

[44] S. Visram, A. S. Bremmer, B. E. Harrington, and G. Hawthorne, "Factors affecting uptake of an education and physical activity programme for newly diagnosed type 2 diabetes," European Diabetes Nursing, vol. 5, no. 1, pp. 17-22, 2008.

[45] M. Mishali, H. Omer, and A. D. Heymann, "The importance of measuring self-efficacy in patients with diabetes," Family Practice, vol. 28, no. 1, pp. 82-87, 2011.

[46] F. Müller-Riemenschneider, T. Reinhold, M. Nocon, and S. N. Willich, "Long-term effectiveness of interventions promoting physical activity: a systematic review," Preventive Medicine, vol. 47, no. 4, pp. 354-368, 2008.

[47] A. P. T. Fayh, A. L. Lopes, P. R. Fernandes, A. ReischakOliveira, and R. Friedman, "Impact of weight loss with or without exercise on abdominal fat and insulin resistance in obese individuals: a randomised clinical trial," British Journal of Nutrition, vol. 110, no. 3, pp. 486-492, 2013.

[48] C. C. Curioni and P. M. Lourenço, "Long-term weight loss after diet and exercise: a systematic review," International Journal of Obesity, vol. 29, no. 10, pp. 1168-1174, 2005.
[49] S. L. Norris, X. Zhang, A. Avenell, E. Gregg, C. H. Schmid, and J. Lau, "Long-term non-pharmacological weight loss interventions for adults with prediabetes," The Cochrane Database of Systematic Reviews, no. 2, Article ID CD005270, 2005.

[50] R. Kouvelioti, G. Vagenas, and S. Langley-Evans, "Effects of exercise and diet on weight loss maintenance in overweight and obese adults: a systematic review," Journal of Sports Medicine and Physical Fitness, vol. 54, no. 4, pp. 456-474, 2014.

[51] R. F. Hamman, R. R. Wing, S. L. Edelstein et al., "Effect of weight loss with lifestyle intervention on risk of diabetes," Diabetes Care, vol. 29, no. 9, pp. 2102-2107, 2006.

[52] A. C. Feldstein, K. Bachman, G. A. Nichols et al., "Weight change in diabetes and glycemic and blood pressure control," Diabetes Care, vol. 31, no. 10, pp. 1960-1965, 2008. 


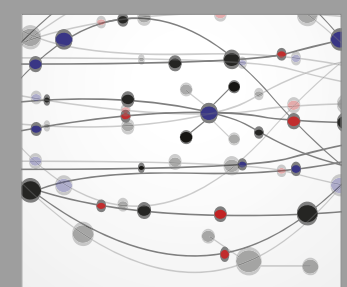

The Scientific World Journal
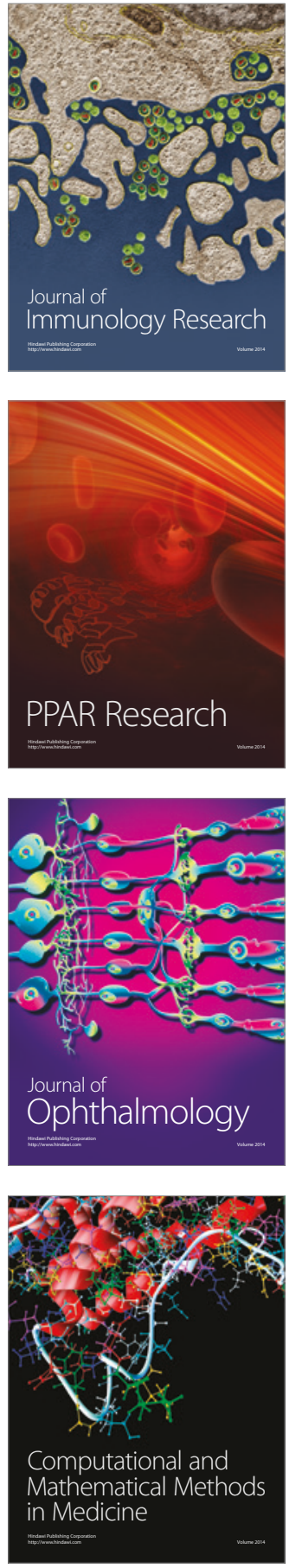

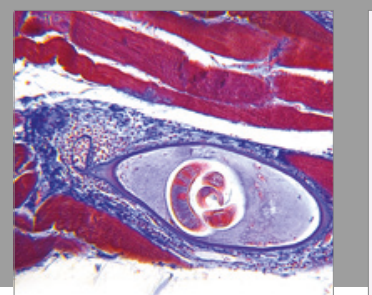

Gastroenterology Research and Practice
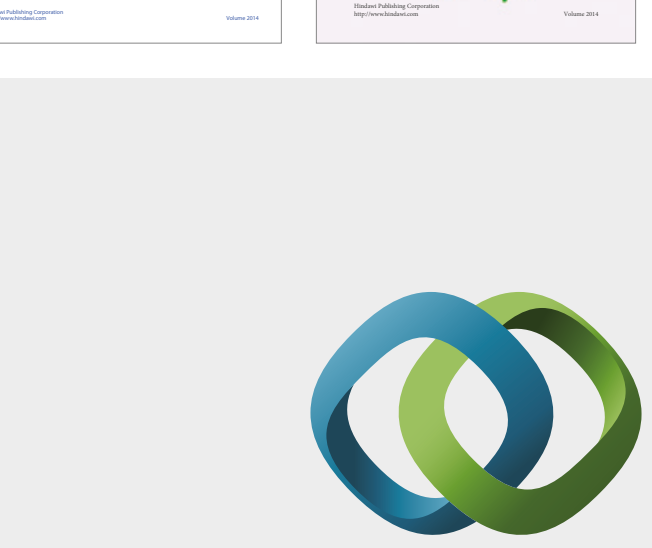

\section{Hindawi}

Submit your manuscripts at

https://www.hindawi.com

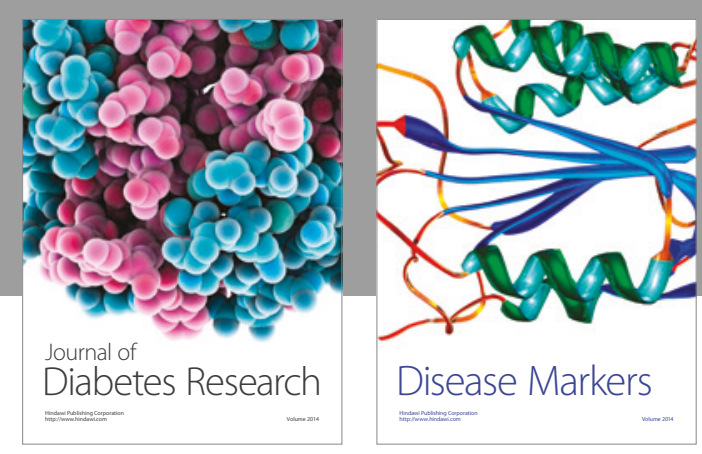

Disease Markers
